\title{
Effects of heme on the thermal stability of mesophilic and thermophilic cytochromes c: Comparison between experimental and theoretical results.
}

\section{AUTHOR(S):}

Oda, Koji; Kodama, Ryota; Yoshidome, Takashi; Yamanaka, Masaru; Sambongi, Yoshihiro; Kinoshita, Masahiro

\section{CITATION:}

Oda, Koji ...[et al]. Effects of heme on the thermal stability of mesophilic and thermophilic cytochromes c: Comparison between experimental and theoretical results.. The Journal of chemical physics 2011, 134(2): 025101.

\section{ISSUE DATE:}

2011-01-14

URL:

http://hdl.handle.net/2433/134794

\section{RIGHT:}

(C) 2011 American Institute of Physics 


\title{
Effects of heme on the thermal stability of mesophilic and thermophilic cytochromes $c$ : Comparison between experimental and theoretical results
}

\author{
Koji Oda, ${ }^{1}$ Ryota Kodama, ${ }^{2}$ Takashi Yoshidome,${ }^{3}$ Masaru Yamanaka, ${ }^{4}$ \\ Yoshihiro Sambongi, ${ }^{4}$ and Masahiro Kinoshita ${ }^{3, a)}$ \\ ${ }^{1}$ Taisho Pharmaceutical Co., Ltd., Yoshino-cho, Kita-ku, Saitama 331-9530, Japan \\ ${ }^{2}$ Graduate School of Energy Science, Kyoto University, Uji, Kyoto 611-0011, Japan \\ ${ }^{3}$ Institute of Advanced Energy, Kyoto University, Uji, Kyoto 611-0011, Japan \\ ${ }^{4}$ Graduate School of Biosphere Science, Hiroshima University, Kagamiyama, Hiroshima 739-8528, Japan
}

(Received 28 June 2010; accepted 3 November 2010; published online 11 January 2011)

\begin{abstract}
We have recently proposed a measure of the thermal stability of a protein: the water-entropy gain at $25^{\circ} \mathrm{C}$ upon folding normalized by the number of residues, which is calculated using a hybrid of the angle-dependent integral equation theory combined with the multipolar water model and the morphometric approach. A protein with a larger value of the measure is thermally more stable. Here we extend the study to analyses on the effects of heme on the thermal stability of four cytochromes $c$ (PA $c_{551}, \mathrm{PH} c_{552}, \mathrm{HT} c_{552}$, and AA $c_{555}$ ) whose denaturation temperatures are considerably different from one another despite that they share significantly high sequence homology and similar threedimensional folds. The major conclusions are as follows. For all the four cytochromes $c$, the thermal stability is largely enhanced by the heme binding in terms of the water entropy. For the holo states, the measure is the largest for AA $c_{555}$. However, AA $c_{555}$ has the lowest packing efficiency of heme and the apo polypeptide with hololike structure, which is unfavorable for the water entropy. The highest stability of AA $c_{555}$ is ascribed primarily to the highest efficiency of side-chain packing of the apo polypeptide itself. We argue for all the four cytochromes $c$ that due to covalent heme linkages, the number of accessible conformations of the denatured state is decreased by the steric hindrance of heme, and the conformational-entropy loss upon folding becomes smaller, leading to an enhancement of the thermal stability. As for the apo state modeled as the native structure whose heme is removed, AA $c_{555}$ has a much larger value of the measure than the other three. Overall, the theoretical results are quite consistent with the experimental observations (e.g., at $25^{\circ} \mathrm{C}$ the $\alpha$-helix content of the apo state of AA $c_{555}$ is almost equal to that of the holo state while almost all helices are collapsed in the apo states of PA $c_{551}, \mathrm{PH} c_{552}$, and HT $c_{552}$ ). (0) 2011 American Institute of Physics. [doi:10.1063/1.3519814]
\end{abstract}

\section{INTRODUCTION}

Bacterial micro-organisms are distributed in a wide range of thermal conditions from subfreezing to over boiling temperatures. Their growth temperature is closely related to the thermal stability of proteins. For instance, the proteins from thermophilic bacteria usually possess higher denaturation temperatures than those from mesophilic bacteria. ${ }^{1,2}$ The proteins exhibiting such various thermal stabilities have been studied rather extensively in experiments. An interesting viewpoint is to compare a specific protein of a thermophilic bacterium with its counterpart homologous protein of a mesophilic bacterium in terms of the structure and the denaturation temperature. ${ }^{3}$ Above all, cytochrome $c$ proteins play essential roles in the electron-transfer reaction in various organisms living under a variety of environmental conditions ranging from low to extremely high temperatures, and they are suitable targets of studies based on the viewpoint mentioned above.

\footnotetext{
a) Author to whom correspondence should be addressed. Electronic mail: kinoshit@iae.kyoto-u.ac.jp.
}

Sambongi and co-workers ${ }^{3-5}$ have experimentally performed comparative studies for cytochrome $c 551$ from Pseudomonas aeruginosa (PA $c_{551}$ ), cytochrome $c 552$ from Pseudomonas hydrogenothermophila ( $\left.\mathrm{PH} c_{552}\right)$, cytochrome $c 552$ from Hydrogenobacter thermophilus (HT $c_{552}$ ), and cytochrome $c 555$ from Aquifex aeolicus (AA $c_{555}$ ). PA $c_{551}$, PH $c_{552}$, HT $c_{552}$, and AA $c_{555}$ are models of mesophilic, moderately thermophilic, thermophilic, and hyperthermophilic proteins, respectively. These proteins are characterized by the heme binding (i.e., covalent heme linkages). Though they share significantly high sequence homology and similar three-dimensional folds (see Fig. 1; the only apparent difference is that AA $c_{555}$ possesses an extra helix), their denaturation temperatures are considerably different: $86.4,108.0$, 121.1 , and $129.7^{\circ} \mathrm{C}$, respectively, at $p \mathrm{H}=5$. The polypeptides with and without the heme binding are referred to as holo and apo states, respectively. In the apo states of PA $c_{551}$, PH $c_{552}$, and HT $c_{552}$, almost all helices are collapsed at $25^{\circ} \mathrm{C} .5,6$ By contrast, the $\alpha$-helix content of the apo state of AA $c_{555}$ is almost equal to that of the holo state. (The $\alpha$-helix content was measured at $p \mathrm{H}=5$ and 7.) The apo AA $c_{555}$ polypeptide can intrinsically form a hololike structure 

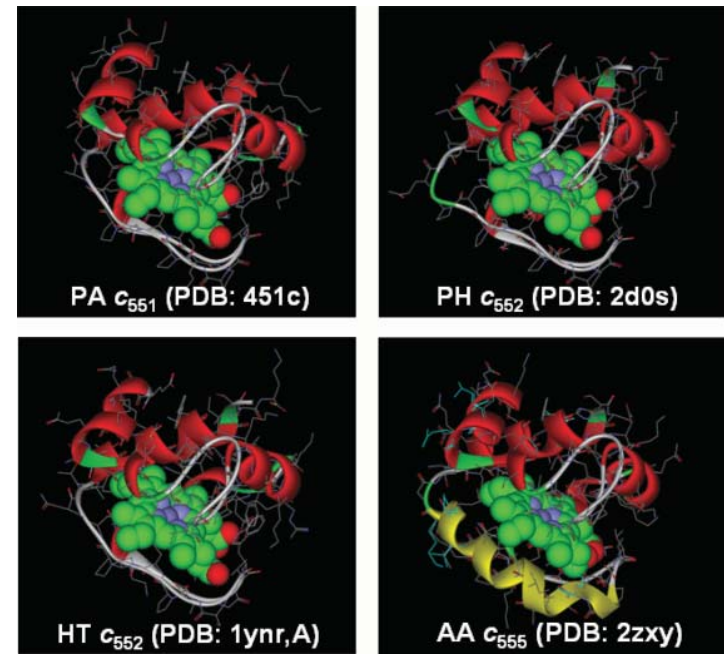

FIG. 1. Native structures of PA $c_{551}, \mathrm{PH} c_{552}$, HT $c_{552}$, and AA $c_{555}$. Ribbon and line representations are employed for apo polypeptides, and CPK models are adopted for heme. AA $c_{555}$ possesses an extra helix marked in yellow. Molecular graphics pictures are prepared using Accelrys Discovery Studio 2.5.5.

without the heme binding. However, the underlying mechanisms of these experimental observations have not been elucidated yet.

We have shown that protein folding is driven by a waterentropy gain. ${ }^{7-11}$ When the $\alpha$-helix or $\beta$-sheet is formed, for example, the excluded volumes generated by the backbone and side chains overlap, leading to an increase in the total volume available to the translational displacement of water molecules that coexist with the protein (i.e., an increase in the number of accessible translational configurations of water) and resulting in an increase in the water entropy. The close packing of side chains, which results in a large water-entropy gain for the same reason, is also crucially important. ${ }^{12}$ The thorough, overall packing of the backbone and side chains with little space in the interior, which is unique to the native structure, is ascribed to this water-entropy effect.

In the conventional concept, ${ }^{13}$ only the water in the close vicinity of a hydrophobic surface is considered when the effects of the water entropy are discussed: The effects are argued primarily in terms of the surface-water orientational correlation, enhanced hydrogen-bonding network of water, and restriction of the rotational freedom of water molecules. We remark that the entropic effect emphasized in the present study (i.e., the excluded-volume effect), which reaches a far larger length scale, ${ }^{7-11}$ is substantially different from the conventionally argued one. Due to the hydrogen bonding water can exist as a dense liquid despite its quite a small molecular size, leading to an exceptionally large entropic effect. It is often claimed that a smaller area of the exposed hydrophobic surface leads to higher thermal stability. This is certainly true. However, the excluded-volume effect is much larger as proved in our earlier publication. ${ }^{14}$ (The conventionally argued effect is also incorporated through our theoretical method described in Sec. II C.)

Protein folding occurs almost under constant-pressure and constant-volume conditions, ${ }^{9}$ and we consider the constant-volume condition which can be handled much more readily in a theoretical study. In our earlier work, ${ }^{14}$ we devel- oped a physical picture of thermal denaturation of proteins. In the picture, thermal denaturation is described by the competition between the water-entropy gain $\Delta S_{\mathrm{VH}}\left(\Delta S_{\mathrm{VH}}>0\right)$ and the loss of protein conformational entropy $\Delta S_{\mathrm{C}}\left(\Delta S_{\mathrm{C}}\right.$ $<0$ ) upon folding. $\Delta S_{\mathrm{VH}}$ and $\left|\Delta S_{\mathrm{C}}\right|$ are, respectively, decreasing and increasing functions of the temperature $T$. The denaturation occurs when $\left|\Delta S_{\mathrm{C}}\right|$ becomes larger than $\Delta S_{\mathrm{VH}}$. We then proposed a measure of the thermal stability: $\Delta S_{\mathrm{VH}}$ calculated at $T=25^{\circ} \mathrm{C}$ (hereafter, this is denoted by $\Delta S_{\mathrm{VH}, 25}$ ) divided by the number of residues $N_{r}$. A protein with a larger value of the measure has higher thermal stability (i.e., a higher denaturation temperature). The validity of the picture was demonstrated for the yeast, bacterial, and human orthologues of frataxins (they are abbreviated to Yfh1, CyaY, and hfra, respectively) whose denaturation temperatures are considerably different: ${ }^{15} 35.9,53.8$, and $60.0^{\circ} \mathrm{C}$ (at $p \mathrm{H}=7.0$ ), respectively, despite that they share significantly high sequence homology and similar three-dimensional folds.

In the present article, we extend our earlier work described above to analyses on the effects of heme on the thermal stability of the four cytochromes $c$, PA $c_{551}, \mathrm{PH} c_{552}$, HT $c_{552}$, and AA $c_{555}$. We investigate the physical origins of the differences among the apo and holo states of PA $c_{551}$, $\mathrm{PH} c_{552}$, HT $c_{552}$, and AA $c_{555}$ in terms of the thermal stability. The physical picture of thermal denaturation is improved in the sense that the temperature dependence of $\left|\Delta S_{\mathrm{C}}\right|$ is described in a more reasonable way. $\Delta S_{\mathrm{VH}, 25} /\left(k_{\mathrm{B}} N_{r}\right)$, which is still a measure of the thermal stability, is calculated using a hybrid of the angle-dependent integral equation theory ${ }^{16-29}$ combined with the multipolar water model ${ }^{17,18}$ and the morphometric approach. ${ }^{30,31}$ Overall, the theoretical results are quite consistent with the experimental observations.

\section{MODEL AND THEORY}

\section{A. Water and protein models}

We employ a multipolar model for water. ${ }^{17,18} \mathrm{~A}$ water molecule is modeled as a hard sphere with diameter $d_{\mathrm{S}}=0.28$ $\mathrm{nm}$ in which a point dipole and a point quadrupole of tetrahedral symmetry are embedded. The effect of the molecular polarizability is taken into account using the self-consistent mean field (SCMF) theory. ${ }^{17,18}$ At the SCMF level the manybody induced interactions are reduced to pairwise additive potentials involving an effective dipole moment.

The hydration entropy $S_{\mathrm{VH}}$ is determined mainly by the excluded-volume effect originating from the translational displacement of water molecules that coexist with a protein in the system and not significantly dependent on the protein-water interaction potentials. Imai et $a .^{32}$ have considered the native structures of a total of eight peptides and proteins and calculated $S_{\mathrm{VH}}$ using the three-dimensional reference interaction site model (3D-RISM) theory combined with the all-atom [Coulomb plus Lennard-Jones (LJ)] potentials and the simple point charge/extended (SPC/E) water model. Even when the protein-water electrostatic potentials, which are quite strong, are shut off and only the LJ potentials are retained, $\left|S_{\mathrm{VH}}\right|$ decreases only by $\sim 5 \%$. Therefore, a protein can be modeled as a set of fused hard spheres. 
Modeling a protein as a set of fused hard spheres can also be justified as follows. The hydration free energy $\mu$, entropy $S_{\mathrm{VH}}$, and energy $U_{\mathrm{VH}}$ under the isochoric condition are calculated for a spherical solute with diameter $0.28 \mathrm{~nm}$ using the angle-dependent integral equation theory ${ }^{16-29}$ combined with the multipolar water model. ${ }^{17,18}$ For the hard-sphere solute with zero charge, the calculated values are $\mu=5.95 k_{\mathrm{B}} T$, $S_{\mathrm{VH}}=-9.22 k_{\mathrm{B}}$, and $U_{\mathrm{VH}}=-3.27 k_{\mathrm{B}} T$. When the point charge $-0.5 e$ ( $e$ is the electronic charge) is embedded at its center, the calculated values are $\mu=-32.32 k_{\mathrm{B}} T, S_{\mathrm{VH}}$ $=-10.11 k_{\mathrm{B}}$, and $U_{\mathrm{VH}}=-42.43 k_{\mathrm{B}} T$. Thus, $S_{\mathrm{VH}}$ is fairly insensitive to the solute-water interaction potential while $\mu$ and $U_{\mathrm{VH}}$ are largely influenced by it.

\section{B. Preparation of protein structures}

For the native structures, the following x-ray structures are taken from Protein Data Bank (PDB): PDB:351c and PDB:451c for PA $c_{551}$, PDB:2d0s for PH $c_{552}$, PDB:1ynr for HT $c_{552}$, and PDB:2zxy for AA $c_{555}$. Some of the PDB entries contain multiple polypeptide chains, and they are split into single chains and treated individually. There are two alternative side-chain conformation models for some residues. We generate as many combinations of the conformation models for different residues as possible to reproduce an ensemble of conformers. For example, PDB:1ynr has four chains: A, B, C, and D. In chains A, C, and D, there are two residues having two alternative side-chain conformation models, and $12\left(=3 \times 2^{2}\right)$ conformers are generated. In chain B, there are four residues having two alternative side-chain conformation models, and $16\left(=2^{4}\right)$ conformers are generated. Thus, we construct a total of 28 conformers for $1 \mathrm{ynr}$. In the case of PDB:2zxy, there is only a single chain but it has nine residues each of which have two alternative side-chain conformation models. Instead of generating all the $512\left(=2^{9}\right)$ conformers, we systematically sample 40 conformers with care so that each model of a residue is impartially sampled. The numbers of the native structures thus constructed are 2, 1, 28, and 40 for PA $c_{551}$, PH $c_{552}$, HT $c_{552}$, and AA $c_{555}$, respectively. In HT $c_{552}, N_{r}=79\left(N_{r}\right.$ is the number of residues resolved in the PDB file) for chains A and C and $N_{r}=80$ for chains B and D. $N_{r}=82,79$, and 86 for PA $c_{551}$, PH $c_{552}$, and AA $c_{555}$, respectively. The native structures are slightly modified using the energy minimization by CHARMM (Ref. 33) to remove unrealistic overlaps of several atoms. Since it is important to keep the original $\mathrm{x}$-ray structures as strictly as possible, we put positional constraints of $50 \mathrm{kcal} / \mathrm{nm}^{2}$ on all the heavy atoms and apply 100 steps of steepest descent and 200 steps of adopted basis Newton-Raphson minimization techniques. The minimization is performed using CHARMM 22 parameters (Ref. 34) with the CMAP correction ${ }^{35}$ and the Generalized Born using Molecular Volume (GBMV) implicit solvent model. ${ }^{36-38}$ The parameters for heme are taken from the publication by Autenrieth and co-workers. ${ }^{39}$

For the unfolded state, we employ five extended structures whose main-chain dihedral angles $(\phi, \psi)$ range from $\left(-130^{\circ}, 130^{\circ}\right)$ to $\left(-170^{\circ}, 170^{\circ}\right)$ by the step of $\left(-10^{\circ}, 10^{\circ}\right)$. For proline, such dihedral angles are not allowed, and $(\phi$, $\psi)$ is set to $\left(-60^{\circ}, 40^{\circ}\right)$. $\omega$ is set to $180^{\circ}$. We take the most probable conformer for each side chain from Dunbrack's Backbone-Dependent Rotamer Library. ${ }^{40,41}$ All the extended structures are slightly modified using the energyminimization technique mentioned above. In cases where heme-bound unfolded structures are needed, we take mainchain and side-chain dihedral angles in the heme-binding region of the native structure: The region comprises eight consecutive residues and fully covers the two cysteine residues binding covalently to heme and the histidine residue coordinating to heme Fe.

In Sec. IV B, we calculate the water-entropy gain upon an imaginary heme binding to examine the packing efficiency of heme and the apo polypeptide with hololike structure. The hololike structures are taken from the native structures, which are constructed as described above, by removing all the heme atoms. Heme is obtained by eliminating all the apo polypeptide atoms. In Sec. IV C, all residues in each of the native and extended structures are replaced by glycine. The replacement is carried out after the energy minimization. The structure thus made, which has essentially no side chains, can be considered as the backbone alone taken from a protein structure. In Sec. IV D, we calculate the number of intramolecular hydrogen bonds inside the apo polypeptide with hololike structure. The hydrogen bonds between heme and the apo polypeptide are excluded in the calculation. In the recognition of a hydrogen bond, we employ the criteria proposed by McDonald and Thornton. ${ }^{42}$ Some of the manipulations and calculations mentioned above are performed with the help of MMSTB Toolkit ${ }^{43}$ and TINKER Program Package. ${ }^{44}$

\section{Hybrid of angle-dependent integral equation theory and morphometric approach}

Since a molecular model is employed for water, the angle-dependent version ${ }^{16-29}$ must be used for the integral equation theory, an elaborate statistical-mechanical theory. However, its extension to complex solute molecules like proteins is rather difficult. This problem can be overcome by combining it with the morphometric approach. ${ }^{30,31}$ The idea of the approach is to express a hydration quantity such as $S_{\mathrm{VH}}$ by the linear combination of only four geometric measures of a solute molecule:

$$
S_{\mathrm{VH}} / k_{\mathrm{B}}=C_{1} V_{\mathrm{ex}}+C_{2} A+C_{3} X+C_{4} Y .
$$

Here, $k_{\mathrm{B}}$ is Boltzmann's constant, $V_{\mathrm{ex}}$ is the excluded volume, $A$ is the water-accessible surface area, and $X$ and $Y$ are the integrated mean and Gaussian curvatures of the accessible surface, respectively. Though $C_{1} V_{\mathrm{ex}}$ is the principal term, the other three terms also influence $S_{\mathrm{VH}}$. In the approach, the solute shape enters $S_{\mathrm{VH}}$ only via the four geometric measures. Therefore, the four coefficients $\left(C_{1}-C_{4}\right)$ can be determined in simple geometries. They are calculated from the values of $S_{\mathrm{VH}}$ for hard-sphere solutes with various diameters immersed in our model water. The angle-dependent integral equation theory ${ }^{16-29}$ is employed in the calculation. Hereafter, the resulting method is referred to as "our hybrid method."

As proved in our earlier work, ${ }^{27}$ the angle-dependent integral equation theory gives a quantitatively accurate value of the hydration free energy of a nonpolar solute. The high 


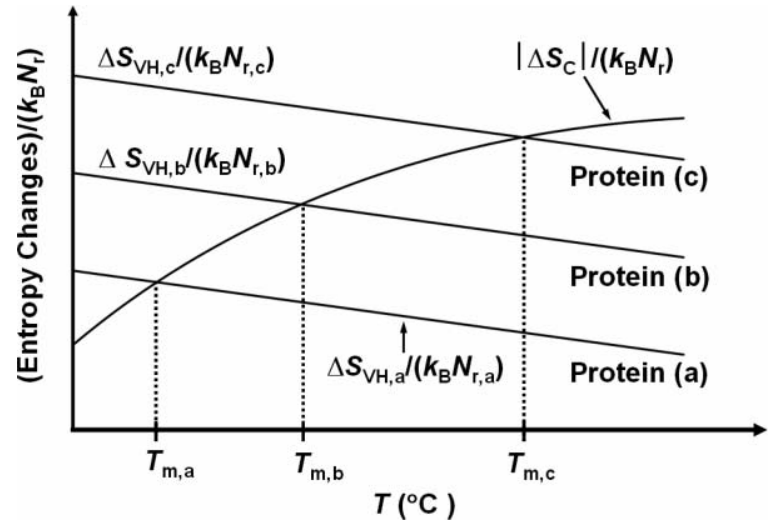

FIG. 2. Simple illustration of our physical picture of thermal denaturation of proteins. Three different proteins, (a), (b), and (c), are considered. $\Delta S_{\mathrm{VH}}$ is the water-entropy gain and $\Delta S_{\mathrm{C}}$ is the conformational-entropy loss upon folding. The subscripts, a, b and c denote the values for proteins (a), (b), and (c), respectively. $\Delta S_{\mathrm{VH}}$ does not necessarily change linearly with the temperature $T$. The three solid lines of $\Delta S_{\mathrm{VH}} /\left(k_{\mathrm{B}} N_{r}\right)$ are not necessarily parallel. $d\left|S_{\mathrm{C}}\right| / d T>0$ and $d^{2}\left|S_{\mathrm{C}}\right| / d T^{2}<0: T_{m, \mathrm{c}}-T_{m, \mathrm{~b}}>T_{m, \mathrm{~b}}-T_{m, \mathrm{a}}$

reliability of the morphometric approach has already been demonstrated. For example, the results from the threedimensional integral equation theory ${ }^{7,8,45-47}$ applied to the same model protein immersed in a simple solvent (the solvent particles interact through strongly attractive potential like water molecules) can be reproduced with sufficient accuracy by the morphometric approach where the four coefficients are determined in the same manner. By our hybrid method, the experimentally measured changes in thermodynamic quantities upon apoplastocyanin folding are quantitatively reproduced. ${ }^{9}$ Further, great progresses have been made in elucidating the microscopic mechanisms of pressure, ${ }^{48,49}$ cold, ${ }^{50,51}$ and heat ${ }^{14}$ denaturating of proteins and in discriminating the native fold from a number of misfolded decoys. ${ }^{52-54}$

\section{PHYSICAL PICTURE OF THERMAL DENATURATION OF PROTEINS}

\section{A. Water-entropy gain and conformational-entropy loss upon protein folding}

Our physical picture of thermal denaturation of proteins is illustrated in Fig. 2. The denaturation temperature is denoted by $T_{m}$. The thorough, overall packing of the backbone and side chains with little space in the interior leads to a substantial decrease in the total excluded volume. ${ }^{7,8,10-12}$ Primarily by this effect, a large gain of the water entropy occurs upon protein folding. This water-entropy gain $\Delta S_{\mathrm{VH}}\left(\Delta S_{\mathrm{VH}}>0\right)$ is dependent on the number density of water $\rho$ and $T$. As $T$ increases with $\rho$ unchanged, $\Delta S_{\mathrm{VH}}$ becomes larger. ${ }^{27}$ However, as $\rho$ decreases with constant $T, \Delta S_{\mathrm{VH}}$ becomes smaller. Above $25^{\circ} \mathrm{C}$, for real water $\rho$ decreases progressively with increasing $T$. As a result, $\Delta S_{\mathrm{VH}}$ is a decreasing function of $T$. In the experiments for thermal denaturation of the four cytochromes $c$, the pressure is set at 10 atm (Refs. 3-5) because except for PA $c_{551} T_{m}$ exceeds the boiling temperature at $1 \mathrm{~atm}$. Elevating the pressure to $10 \mathrm{~atm}$ causes only a slight increase in the number density of water, which necessitates no consideration of the resultant effect.
Protein folding accompanies a large loss of the protein conformational entropy $\Delta S_{\mathrm{C}}\left(\Delta S_{\mathrm{C}}<0\right)$. Hereafter, $S_{\mathrm{C}, \mathrm{N}}$ is the conformational entropy of the native structure and $S_{\mathrm{C}, \mathrm{D}}$ is that of the denatured state. (The subscripts "N" and "D" denote "native" and "denatured," respectively, and $\Delta S_{\mathrm{C}}$ $=S_{\mathrm{C}, \mathrm{N}}-S_{\mathrm{C}, \mathrm{D}}$.) $S_{\mathrm{C}, \mathrm{N}}$ remains roughly the same against a temperature increase due to the constraints caused by the closely packed structure, while this is not the case for $S_{\mathrm{C}, \mathrm{D}} . S_{\mathrm{C}, \mathrm{D}}$ is closely related to the allowed ranges of dihedral angles which are dependent on the torsion energy and $T$. Angles giving high torsion energy are not allowed at a low temperature. As $T$ increases, the allowed range of each angle becomes increasingly wider, leading to larger $S_{\mathrm{C}, \mathrm{D}}$. As $T$ increases further, the enlargement of $S_{\mathrm{C}, \mathrm{D}}$ is decelerated due to the steric repulsion among atoms in a residue and among atoms in neighboring residues. It follows that for the denatured state $d S_{\mathrm{C}, \mathrm{D}} / d T>0$ and $d^{2} S_{\mathrm{C}, \mathrm{D}} / d T^{2}<0$ and that $\left|\Delta S_{\mathrm{C}}\right|$ exhibits the same temperature dependence $\left(d\left|\Delta S_{\mathrm{C}}\right| / d T>0\right.$ and $\left.d^{2}\left|\Delta S_{\mathrm{C}}\right| / d T^{2}<0\right)$. This argument is consistent with the experimental result reported by Fitter. ${ }^{55}$

We treat homologous proteins. A protein consists of a number of amino-acid residues. The usage of the 20 aminoacid species does not largely differ from protein to protein. Therefore, in general $S_{\mathrm{C}, \mathrm{D}}$ is almost in proportion to the number of residues $N_{r}$ while $S_{\mathrm{C}, \mathrm{N}}$ is essentially zero, and $\left|\Delta S_{\mathrm{C}}\right|$ is approximately proportionate to $N_{r}$. Further, all the proteins share roughly the same proportional constant and $\left|\Delta S_{\mathrm{C}}\right| /\left(k_{\mathrm{B}} N_{r}\right)$ can be considered to be independent of the protein species. This cannot be applied to proteins with $\mathrm{S}-\mathrm{S}$ bonds because the conformational entropy of the denatured state is significantly reduced by the $\mathrm{S}-\mathrm{S}$ bonds.

For homologous proteins, $S_{\mathrm{VH}} / k_{\mathrm{B}}$ of the denatured state divided by $N_{r}, S_{\mathrm{VH}, \mathrm{D}} /\left(k_{\mathrm{B}} N_{r}\right)$, is also almost independent of the protein species. However, this is not true for $S_{\mathrm{VH}, \mathrm{N}} /\left(k_{\mathrm{B}} N_{r}\right)$ that is quite sensitive to the manner of backbone and sidechain packing in the native structure. As a consequence, $\Delta S_{\mathrm{VH}} /\left(k_{\mathrm{B}} N_{r}\right)\left(\Delta S_{\mathrm{VH}}=S_{\mathrm{VH}, \mathrm{N}}-S_{\mathrm{VH}, \mathrm{D}}\right)$ varies considerably from protein to protein: $\Delta S_{\mathrm{VH}} /\left(k_{\mathrm{B}} N_{r}\right)$ is largely influenced by the compactness of the native structure and tightness of the packing of the backbone and side chains.

\section{B. Measure of thermal stability of proteins}

In our physical picture illustrated in Fig. 2, the thermal stability is described by the competition of the water-entropy gain per residue and the conformational-entropy loss per residue. ${ }^{14}$ Below $T_{m}, \Delta S_{\mathrm{VH}} /\left(k_{\mathrm{B}} N_{r}\right)$ is larger than $\left|\Delta S_{\mathrm{C}}\right| /\left(k_{\mathrm{B}} N_{r}\right)$ and the native structure is more stable. Above $T_{m}$, the inversion occurs, leading to thermal denaturation. Three different proteins, (a), (b), and (c), are considered in Fig. 2. We consider $\Delta S_{\mathrm{VH}, 25} /\left(k_{\mathrm{B}} N_{r}\right)$ as a measure of the thermal stability of a protein. The larger the measure is, the higher $T_{m}$ is. The slopes of the three solid lines for proteins (a), (b), and (c) can be different, but the difference is assumed to be not large enough to invert the order of the thermal stability. Because of $d^{2}\left|\Delta S_{\mathrm{C}}\right| / d T^{2}<0$, the difference in $T_{m}$ between proteins (b) and (c) is larger than that between proteins (a) and (b) even when the differences in the measure between proteins (b) and (c) and between proteins (a) and (b) share the same value. 
For proteins with very high denaturation temperatures like the four cytochromes $c$, only a small difference in the measure can lead to a large difference in $T_{m}$.

Protein folding accompanies a change in energy $\Delta U$ as well. It is experimentally known that $\Delta U$ decreases with increasing $T$ and takes a negative value at $T=T_{m} .{ }^{56}$ This can be understood as follows. When a protein folds, donors and acceptors (e.g., $\mathrm{NH}$ and $\mathrm{C}=\mathrm{O}$, respectively) are buried in the interior after the break of hydrogen bonds with water molecules ( $\mathrm{NH} \cdots \mathrm{W}, \mathrm{C}=\mathrm{O} \cdots \mathrm{W}$, etc.). They form as many intramolecular hydrogen bonds as possible to make up for the penalty arising from the loss of hydrogen bonds with water molecules (i.e., the dehydration penalty). As $T$ increases, due to more energetic thermal motion of water molecules, the hydrogen bonds with them become less stable and the dehydration penalty decreases with increasing $T$. On the other hand, the energy lowering provided by the intramolecular hydrogen bonding remains roughly unchanged. $\Delta U$ is negative at $T$ $=T_{m}$, and the native structure is more stable than the unfolded state in terms of energy. Thus, thermal denaturation cannot be driven energetically. Our concern is just to determine the order in terms of the thermal stability of some different proteins. The incorporation of $\Delta U /\left\{k_{\mathrm{B}}(T+273.15) N_{r}\right\}$ in the physical picture will shift the value of $T_{m}$, but the order is hopefully unchanged. Hereafter, we discuss the thermal stability of some homologous proteins by comparing $\Delta S_{\mathrm{VH}, 25} /\left(k_{\mathrm{B}} N_{r}\right)$. The effect of the energetic component is discussed in Sec. IV D.

\section{Structural model of unfolded state}

In our earlier work, ${ }^{14}$ we adopted random coils for the unfolded state of the yeast, bacterial, and human orthologues of frataxins. When random coils are adopted, however, we have to consider many different random coils. Moreover, with the covalent heme linkages the generation of random coils cannot readily be done. For these reasons, we employ extended structures as the unfolded state of the four cytochromes $c$. In order to examine the validity of this employment, we calculate $\Delta S_{\mathrm{VH}, 25} /\left(k_{\mathrm{B}} N_{r}\right)$ for Yfh1 (PDB: 2ga5), CyaY (PDB: 1soy), and hfra (PDB: 11y7). ${ }^{15} \Delta S_{\mathrm{VH}, 25, \mathrm{RC}} /\left(k_{\mathrm{B}} N_{r}\right)$ and $\Delta S_{\mathrm{VH}, 25, \mathrm{EX}} /\left(k_{\mathrm{B}} N_{r}\right)$ are compared in Table I. The subscripts "RC" and "EX," respectively, denote the measures calculated for the transition from a sufficiently large set of random coils to the native structure and for the transition from an extended structure (see Sec. II B) to the native structure. The method of generating the random coils is described in our earlier publication. ${ }^{14}$ In the calculation of $\Delta S_{\mathrm{VH}, 25, \mathrm{RC}} /\left(k_{\mathrm{B}} N_{r}\right)$, the hydration entropy of the random-coil state is taken to be the averaged value for the set of random coils generated (the hydration entropy varies largely from random coil to random coil). The orders of $\Delta S_{\mathrm{VH}, 25, \mathrm{RC}} /\left(k_{\mathrm{B}} N_{r}\right)$ and $\Delta S_{\mathrm{VH}, 25, \mathrm{EX}} /\left(k_{\mathrm{B}} N_{r}\right)$ are both in accord with the order of the denaturation temperature. As mentioned above, $\Delta S_{\mathrm{VH}, 25, \mathrm{EX}} /\left(k_{\mathrm{B}} N_{r}\right)$ is much more convenient as the measure from the viewpoint of the computational load, and we employ $\Delta S_{\mathrm{VH}, 25, \mathrm{EX}} /\left(k_{\mathrm{B}} N_{r}\right)$ as the measure. This implies that an extend structure, whose hydration
TABLE I. Measures of the thermal stability, $\Delta S_{\mathrm{VH}, 25, \mathrm{RC}} /\left(k_{\mathrm{B}} N_{r}\right)$ and $\Delta S_{\mathrm{VH}, 25, \mathrm{EX}} /\left(k_{\mathrm{B}} N_{r}\right)$, for $\mathrm{Yfh} 1, \mathrm{CyaY}$, and hfra. The subscripts "RC" and "EX," respectively, denote the measures calculated for the transition from a sufficiently large set of random coils to the native structure and for the transition from an extended structure to the native structure. " $\Delta S_{\mathrm{VH}, 25, \mathrm{EX}} /\left(k_{\mathrm{B}} N_{r}\right)$ (130)", for example, represents $\Delta S_{\mathrm{VH}, 25, \mathrm{EX}} /\left(k_{\mathrm{B}} N_{r}\right)$ calculated for an extended structure whose main-chain dihedral angles $(\phi, \psi)$ are $\left(-130^{\circ}, 130^{\circ}\right)$.

\begin{tabular}{lccc}
\hline \hline & Yfh1 & CyaY & hfra \\
\hline$T_{m}\left({ }^{\circ} \mathrm{C}\right)$ & 35.9 & 53.8 & 60.0 \\
$N_{r}$ & 123 & 106 & 121 \\
$\Delta S_{\mathrm{VH}, 25, \mathrm{RC}} /\left(k_{\mathrm{B}} N_{r}\right)$ & 3.43 & 3.83 & 5.80 \\
$\Delta S_{\mathrm{VH}, 25, \mathrm{EX}} /\left(k_{\mathrm{B}} N_{r}\right)(130)$ & 5.13 & 5.58 & 7.86 \\
$\Delta S_{\mathrm{VH}, 25, \mathrm{EX}} /\left(k_{\mathrm{B}} N_{r}\right)(140)$ & 4.89 & 5.40 & 7.77 \\
$\Delta S_{\mathrm{VH}, 25, \mathrm{EX}} /\left(k_{\mathrm{B}} N_{r}\right)(150)$ & 4.85 & 5.34 & 7.85 \\
$\Delta S_{\mathrm{VH}, 25, \mathrm{EX}} /\left(k_{\mathrm{B}} N_{r}\right)(160)$ & 4.77 & 5.39 & 7.84 \\
$\Delta S_{\mathrm{VH}, 25, \mathrm{EX}} /\left(k_{\mathrm{B}} N_{r}\right)(170)$ & 4.40 & 4.98 & 7.56 \\
$\Delta S_{\mathrm{VH}, 25, \mathrm{EX}} /\left(k_{\mathrm{B}} N_{r}\right)($ average $)$ & 4.81 & 5.34 & 7.78 \\
\hline \hline
\end{tabular}

entropy is nearly the largest, is considered as the reference structure.

\section{RESULTS AND DISCUSSION}

\section{A. Thermal stability of holo state}

We calculate the measure $\Delta S_{\mathrm{VH}, 25, \mathrm{EX}} /\left(k_{\mathrm{B}} N_{r}\right)$ for the transition from an extended structure to the native structure (see Fig. 3) for the four cytochromes $c$ : PA $c_{551}$, PH $c_{552}$, HT $c_{552}$, and AA $c_{555}$. In the extended structure, heme is covalently bound to the polypeptide. The result of the calculation is given in Table II. The order of the measure is in accord with that of $T_{m}$, PA $c_{551}<\mathrm{PH} c_{552}<\mathrm{HT} c_{552}<\mathrm{AA} c_{555}$, though the differences among the values of $\Delta S_{\mathrm{VH}, 25, \mathrm{EX}} /\left(k_{\mathrm{B}} N_{r}\right)$ for PA $c_{551}$, $\mathrm{PH} c_{552}$, and HT $c_{552}$ are rather small. We note that even a small difference in the measure can lead to a significantly large difference in $T_{m}$ due to $d^{2}\left|\Delta S_{\mathrm{C}}\right| / d T^{2}<0$ (see Fig. 2).

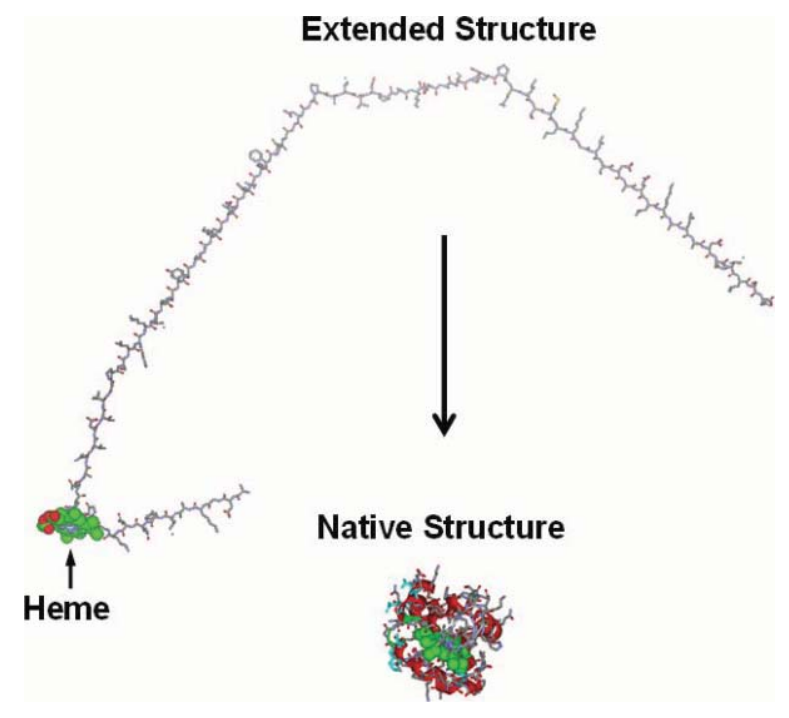

FIG. 3. Transition from an extended structure to the native structure for the holo state. In the extended structure, heme is covalently bound to the apo polypeptide. Molecular graphics pictures are prepared using Accelrys Discovery Studio 2.5.5. 
TABLE II. Measure of the thermal stability $\Delta S_{\mathrm{VH}, 25, \mathrm{EX}} /\left(k_{\mathrm{B}} N_{r}\right)$ for the transition from an extended structure to the native structure (see Fig. 3) for holo states of PA $c_{551}, \mathrm{PH} c_{552}, \mathrm{HT} c_{552}$, and AA $c_{555} \cdot "{ } \Delta S_{\mathrm{VH}, 25, \mathrm{EX}} /\left(k_{\mathrm{B}} N_{r}\right)$ (130)," for example, represents $\Delta S_{\mathrm{VH}, 25, \mathrm{EX}} /\left(k_{\mathrm{B}} N_{r}\right)$ calculated for an extended structure whose main-chain dihedral angles $(\phi, \psi)$ are $\left(-130^{\circ}\right.$, $130^{\circ}$ ). In HT $c_{552}, N_{r}=79$ for chains A and C and $N_{r}=80$ for chains $\mathrm{B}$ and $\mathrm{D}$.

\begin{tabular}{lcccc}
\hline \hline & PA $c_{551}$ & PH $c_{552}$ & HT $c_{552}$ & AA $c_{555}$ \\
\hline$T_{m}\left({ }^{\circ} \mathrm{C}\right)$ & 86.4 & 108.0 & 121.1 & 129.7 \\
$N_{r}$ & 82 & 79 & 79,80 & 86 \\
$\Delta S_{\mathrm{VH}, 25, \mathrm{EX}} /\left(k_{\mathrm{B}} N_{r}\right)(130)$ & 9.18 & 9.25 & 9.42 & 9.44 \\
$\Delta S_{\mathrm{VH}, 25, \mathrm{EX}} /\left(k_{\mathrm{B}} N_{r}\right)(140)$ & 9.01 & 9.18 & 9.18 & 9.51 \\
$\Delta S_{\mathrm{VH}, 25, \mathrm{EX}} /\left(k_{\mathrm{B}} N_{r}\right)(150)$ & 8.95 & 9.03 & 9.06 & 9.53 \\
$\Delta S_{\mathrm{VH}, 25, \mathrm{EX}} /\left(k_{\mathrm{B}} N_{r}\right)(160)$ & 8.78 & 8.96 & 8.99 & 9.38 \\
$\Delta S_{\mathrm{VH}, 25, \mathrm{EX}} /\left(k_{\mathrm{B}} N_{r}\right)(170)$ & 8.23 & 8.55 & 8.59 & 9.27 \\
$\Delta S_{\mathrm{VH}, 25, \mathrm{EX}} /\left(k_{\mathrm{B}} N_{r}\right)($ average $)$ & 8.83 & 8.99 & 9.05 & 9.43 \\
\hline \hline
\end{tabular}

Though we are not sure of the relative accuracy of the values of $\Delta S_{\mathrm{VH}, 25, \mathrm{EX}} /\left(k_{\mathrm{B}} N_{r}\right)$ for PA $c_{551}, \mathrm{PH} c_{552}$, and HT $c_{552}$, we can conclude that AA $c_{555}$ has the highest value of the measure.

Here we remark that the result in Table II cannot be compared with that in Table I in a straightforward manner. The yeast, bacterial, and human orthologues of frataxins are not homologous with the four cytochromes $c$. Therefore, $\left|\Delta S_{\mathrm{C}}\right| /\left(k_{\mathrm{B}} N_{r}\right)$ for the latter can differ significantly from that for the former. Moreover, in the case of the four cytochromes $c$, the covalent heme linkages reduce $\left|\Delta S_{\mathrm{C}}\right| /\left(k_{\mathrm{B}} N_{r}\right)$ to a considerable extent as argued in Sec. IV E.

\section{B. Packing efficiency of heme and apo polypeptide with hololike structure}

The order of the thermal stability observed for holo states may be attributable to that of the packing efficiency of heme and the apo polypeptide with hololike structure. To examine the packing efficiency, we calculate the water-entropy gain normalized by the number of residues upon the imaginary heme binding, $\Delta S_{\mathrm{VH}, 25, \mathrm{HB}} /\left(k_{\mathrm{B}} N_{r}\right)$ (see Fig. 4). We note that $\Delta S_{\mathrm{VH}, 25, \mathrm{HB}} /\left(k_{\mathrm{B}} N_{r}\right)$ increases as the packing efficiency becomes higher. In addition, we calculate the atom-atom contact number between heme and the apo polypeptide with hololike structure $N_{\mathrm{C}}$ normalized by $N_{r}, N_{\mathrm{C}} / N_{r}$. Hydrogen atoms are not treated in the calculation. Atoms $i$ and $j$ are considered to be in contact with each other when the distance between their centers $r_{i j}$ is smaller than $\xi\left(\sigma_{i}+\sigma_{j}\right) / 2\left(\sigma_{k}\right.$ is the Lennard-Jones sigma-value of atom $k$ ). Even when $r_{i j}$ is larger than $\left(\sigma_{i}+\sigma_{j}\right) / 2$, if $\left\{r_{i j}-\left(\sigma_{i}+\sigma_{j}\right) / 2\right\}$ is smaller than the diameter of water molecules $\left(d_{\mathrm{S}}=0.28 \mathrm{~nm}\right)$, a decrease in the excluded volume for water molecules occurs, leading to a water-entropy gain. $\xi$ is chosen so that $\left(\sigma_{i}+\sigma_{j}\right) / 2<\xi\left(\sigma_{i}\right.$ $\left.+\sigma_{j}\right) / 2<\left(\sigma_{i}+\sigma_{j}\right) / 2+d_{\mathrm{S}}$ is satisfied: The three values, 1.3 , 1.5 , and 1.7 , are tested for $\xi . N_{\mathrm{C}} / N_{r}$ should be somewhat correlated with the packing efficiency. The result is given in Table III. Counterintuitively, $\Delta S_{\mathrm{VH}, 25, \mathrm{HB}} /\left(k_{\mathrm{B}} N_{r}\right)$ is the smallest for AA $c_{555} . N_{\mathrm{C}} / N_{r}$ is also the smallest for AA $c_{555}$. The highest thermal stability of the holo state of AA $c_{555}$ is not

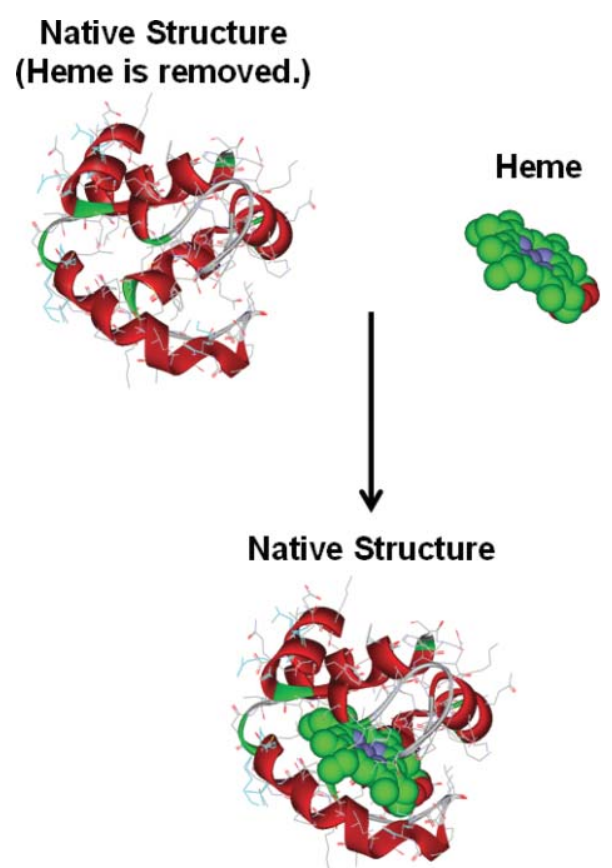

FIG. 4. Imaginary binding of heme to the apo polypeptide with hololike structure. The hololike structure is taken from the native structure by removing all the heme atoms. Heme is obtained by eliminating all the apo polypeptide atoms. Molecular graphics pictures are prepared using Accelrys Discovery Studio 2.5.5.

attributable to the packing efficiency of heme and the apo polypeptide with hololike structure (it is ascribed to the highest stability of its apo state as discussed in Sec. IV C).

Cytochrome $c$ is characterized by covalent heme linkages via thioether bonds between heme vinyl groups and two Cys residues within the consensus heme-binding $\mathrm{CXXCH}$ motif of the polypeptide. Sambongi and co-workers ${ }^{5}$ mutated the Cys residues of AA $c_{555}$ and examined the resultant variant (hereafter, this variant is referred to as $\mathrm{C} 12 \mathrm{~A} / \mathrm{C} 15 \mathrm{~A}$ ) which no longer covalently bound heme via thioether bonds. The experimental result is the following. The $\alpha$-helix contents of holo and apo states of $\mathrm{C} 12 \mathrm{~A} / \mathrm{C} 15 \mathrm{~A}$ are almost the same $(59 \%$ and $57 \%$, respectively) and not significantly different from the $\alpha$ helix content of holo state of AA $c_{555}(61 \%)$. This is indicative that the loss of the thioether bonds and removal of heme do not affect the structure of AA $c_{555}$. The apo AA $c_{555}$ polypeptide intrinsically forms a hololike structure without the heme binding. ${ }^{5}$ This is in marked contrast with the apo PA $c_{551}$, $\mathrm{PH} c_{552}$, and $\mathrm{HT} c_{552}$ polypeptides in which almost all helices

TABLE III. Water-entropy gain normalized by $N_{r}$ upon the heme binding (see Fig. 4), $\Delta S_{\mathrm{VH}, 25, \mathrm{HB}} /\left(k_{\mathrm{B}} N_{r}\right)$, and atom-atom contact number between heme and hololike structure of apo polypeptide $N_{\mathrm{C}}$ normalized by $N_{r}, N_{\mathrm{C}} / N_{r}$, for PA $c_{551}, \mathrm{PH} c_{552}, \mathrm{HT} c_{552}$, and AA $c_{555}$. " $N_{\mathrm{C}} / N_{r}$ (1.3)," for example, represents $N_{\mathrm{C}} / N_{r}$ for $\xi=1.3$.

\begin{tabular}{lcccc}
\hline \hline & PA $c_{551}$ & PH $c_{552}$ & HT $c_{552}$ & AA $c_{555}$ \\
\hline$\Delta S_{\mathrm{VH}, 25, \mathrm{HB}} /\left(k_{\mathrm{B}} N_{r}\right)$ & 2.63 & 2.66 & 2.55 & 2.28 \\
$N_{\mathrm{C}} / N_{r}(1.3)$ & 1.30 & 1.22 & 1.28 & 1.16 \\
$N_{\mathrm{C}} / N_{r}(1.5)$ & 1.72 & 1.77 & 1.83 & 1.66 \\
$N_{\mathrm{C}} / N_{r}(1.7)$ & 2.26 & 2.25 & 2.34 & 2.17 \\
\hline
\end{tabular}




\section{Extended Structure}

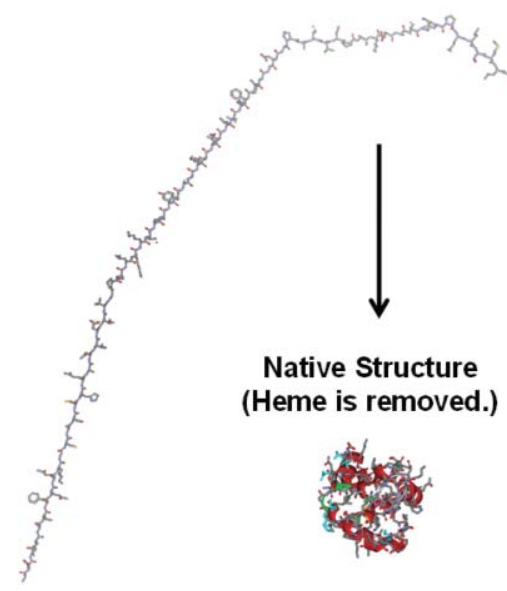

FIG. 5. Imaginary transition from an extended structure of apo polypeptide to the native structure whose heme is removed. In this transition, heme is not incorporated. Molecular graphics pictures are prepared using Accelrys Discovery Studio 2.5.5.

are collapsed. ${ }^{5,6}$ Thus, our theoretical result is quite consistent with the experimental one.

\section{Packing efficiency of apo polypeptide itself}

The result described in Sec. IV B is indicative that the packing efficiency of the apo polypeptide itself plays essential roles in the thermal stability of the holo state. In order to examine the packing efficiency of the apo polypeptide, we calculate the measure $\Delta S_{\mathrm{VH}, 25, \mathrm{EX}} /\left(k_{\mathrm{B}} N_{r}\right)$ for the imaginary transition illustrated in Fig. 5 from an extended structure of the apo polypeptide to the native structure whose heme is removed (i.e., our model apo state). That is, heme is not incorporated in the calculation. The result is given in Table IV. It is observed that the order of $T_{m}$ for model apo states is in accord with that for holo states (see Table II). The measure in the case without heme is considerably smaller than that in the case with heme for all the four cytochromes $c$. This result is consistent with the experimental evidence ${ }^{3-6}$ that the thermal stability of the apo state is much lower than that of the holo state. In the case without heme the measure is much larger for AA $c_{555}$ than for the other three. This result must closely be

TABLE IV. Measure of the thermal stability $\Delta S_{\mathrm{VH}, 25, \mathrm{EX}} /\left(k_{\mathrm{B}} N_{r}\right)$ for the transition from an extended structure to the native structure (see Fig. 5) for apo states of PA $c_{551}$, PH $c_{552}$, HT $c_{552}$, and AA $c_{555} \cdot " \Delta S_{\mathrm{VH}, 25, \mathrm{EX}} /\left(k_{\mathrm{B}} N_{r}\right)$ (130)," for example, represents $\Delta S_{\mathrm{VH}, 25, \mathrm{EX}} /\left(k_{\mathrm{B}} N_{r}\right)$ calculated for an extended structure whose main-chain dihedral angles $(\phi, \psi)$ are $\left(-130^{\circ}\right.$, $\left.130^{\circ}\right)$.

\begin{tabular}{lcccc}
\hline \hline & PA $c_{551}$ & PH $c_{552}$ & HT $c_{552}$ & AA $c_{555}$ \\
\hline$\Delta S_{\mathrm{VH}, 25, \mathrm{EX}} /\left(k_{\mathrm{B}} N_{r}\right)(130)$ & 7.23 & 7.32 & 7.60 & 7.78 \\
$\Delta S_{\mathrm{VH}, 25, \mathrm{EX}} /\left(k_{\mathrm{B}} N_{r}\right)(140)$ & 6.98 & 7.21 & 7.37 & 7.78 \\
$\Delta S_{\mathrm{VH}, 25, \mathrm{EX}} /\left(k_{\mathrm{B}} N_{r}\right)(150)$ & 6.92 & 7.00 & 7.22 & 7.86 \\
$\Delta S_{\mathrm{VH}, 25, \mathrm{EX}} /\left(k_{\mathrm{B}} N_{r}\right)(160)$ & 6.68 & 6.90 & 7.05 & 7.70 \\
$\Delta S_{\mathrm{VH}, 25, \mathrm{EX}} /\left(k_{\mathrm{B}} N_{r}\right)(170)$ & 6.42 & 6.77 & 6.71 & 7.57 \\
$\Delta S_{\mathrm{VH}, 25, \mathrm{EX}} /\left(k_{\mathrm{B}} N_{r}\right)($ average $)$ & 6.85 & 7.04 & 7.19 & 7.74 \\
\hline \hline
\end{tabular}

TABLE V. Decomposition of $\Delta S_{\mathrm{VH}, 25, \mathrm{EX}} /\left(k_{\mathrm{B}} N_{r}\right)$ in Table IV into contributions from the backbone packing $\Delta S_{\mathrm{B}} /\left(k_{\mathrm{B}} N_{r}\right)$ and from the side-chain packing $\Delta S_{\mathrm{S}} /\left(k_{\mathrm{B}} N_{r}\right)$. For an extended structure we consider main-chain dihedral angles of $(\phi, \psi)=\left(-150^{\circ}, 150^{\circ}\right)$.

\begin{tabular}{lcccc}
\hline \hline & PA $c_{551}$ & PH $c_{552}$ & HT $c_{552}$ & AA $c_{555}$ \\
\hline$\Delta S_{\mathrm{VH}, 25, \mathrm{EX}} /\left(k_{\mathrm{B}} N_{\mathrm{r}}\right)$ & 6.92 & 7.00 & 7.22 & 7.86 \\
$\Delta S_{\mathrm{B}} /\left(k_{\mathrm{B}} N_{\mathrm{r}}\right)$ & 1.23 & 1.22 & 1.19 & 1.19 \\
$\Delta S_{\mathrm{S}} /\left(k_{\mathrm{B}} N_{\mathrm{r}}\right)$ & 5.69 & 5.78 & 6.03 & 6.67 \\
\hline \hline
\end{tabular}

related to the following experimental observation: ${ }^{3-6}$ In the apo states of PA $c_{551}$, PH $c_{552}$, and HT $c_{552}$, almost all helices are collapsed at $25^{\circ} \mathrm{C}$ whereas the $\alpha$-helix content of the apo state of AA $c_{555}$ is almost equal to that of the holo state. The structures of real apo states in aqueous solution can be different from those assumed in the present study. However, we are concerned not with the order of the thermal stability of apo states but with the roles of the apo polypeptide in the stabilization of holo states.

We calculate the hydration entropy for a protein with a prescribed structure, quantity 1 . We also calculate it for the same protein whose residues are all replaced by Gly, quantity 2. Quantity 2 can be regarded as the contribution from the backbone to the hydration entropy and "quantity 1 minus quantity 2" is the contribution from side chains to it. For the transition from an extended structure of the apo polypeptide to the native structure whose heme is removed (see Fig. 5), $\Delta S_{\mathrm{VH}, 25, \mathrm{EX}} /\left(k_{\mathrm{B}} N_{r}\right)$ is decomposed into the contribution from the backbone packing to the water-entropy gain upon the transition, $\Delta S_{\mathrm{B}} /\left(k_{\mathrm{B}} N_{r}\right)$, and the contribution from the side-chain packing to the water-entropy gain upon the transition, $\Delta S_{\mathrm{S}} /\left(k_{\mathrm{B}} N_{r}\right)$. For an extended structure we consider main-chain dihedral angles of $(\phi, \psi)=\left(-150^{\circ}, 150^{\circ}\right)$. $\Delta S_{\mathrm{B}} /\left(k_{\mathrm{B}} N_{r}\right)$ is obtained as "quantity 2 of the native structure" minus "quantity 2 of an extended structure," and $\Delta S_{\mathrm{S}} /\left(k_{\mathrm{B}} N_{r}\right)$ $=\Delta S_{\mathrm{VH}, 25, \mathrm{EX}} /\left(k_{\mathrm{B}} N_{r}\right)-\Delta S_{\mathrm{B}} /\left(k_{\mathrm{B}} N_{r}\right) . \quad \Delta S_{\mathrm{B}} /\left(k_{\mathrm{B}} N_{r}\right) \quad$ and $\Delta S_{\mathrm{S}} /$ $\left(k_{\mathrm{B}} N_{r}\right)$ increase as the packing efficiency of the backbone and that of side chains become higher, respectively. The values of $\Delta S_{\mathrm{B}} /\left(k_{\mathrm{B}} N_{r}\right)$ and $\Delta S_{\mathrm{S}} /\left(k_{\mathrm{B}} N_{r}\right)$ for the four cytochromes $c$ are collected in Table V. The packing efficiency of the backbone is roughly the same, but that of side chains is the highest for AA $c_{555}$, making its apo state the most stable.

\section{Effect of energetic component on thermal stability}

As described above, at $T=T_{m}$ protein unfolding leads to an increase in energy. This is principally because at high temperatures the increase in energy due to the loss of protein intramolecular hydrogen bonds is larger than the decrease in energy arising from the formation of protein-water intermolecular hydrogen bonds. The intramolecular-energy increase upon unfolding normalized by $N_{r}$ could contribute to the thermal stability. For example, AA $c_{555}$ possesses an extra helix and the largest number of intramolecular hydrogen bonds $N_{\mathrm{HB}}$, which could be partly responsible for its highest stability. Since the effect of intramolecular hydrogen bonds enters not through $N_{\mathrm{HB}}$ but through $N_{\mathrm{HB}} / N_{r}$, we examine the latter: The values of $N_{\mathrm{HB}} / N_{r}$ for PA $c_{551}, \mathrm{PH} c_{552}, \mathrm{HT} c_{552}$, 
and AA $c_{555}$ are $0.76,0.73,0.77$, and 0.77 , respectively. There is no appreciable correlation between $N_{\mathrm{HB}} / N_{r}$ and $T_{m}$. The intramolecular hydrogen bonds in the extra helix of AA $c_{555}$ make no significant contribution to the highest thermal stability.

\section{E. Effect of heme on conformational-entropy loss upon protein folding}

For all the four cytochromes $c$, the thermal stability is enhanced by the heme binding in terms of the water entropy. We believe that there is an additional factor which enhances the stability with heme: Due to covalent heme linkages, the number of accessible conformations of the denatured state is decreased by the steric hindrance of heme, and the conformational-entropy loss upon folding is reduced (the four cytochromes $c$ share almost the same degree of the reduction). According to the experimental measurements using $\mathrm{C} 12 \mathrm{~A} / \mathrm{C} 15 \mathrm{~A}, T_{m}$ of the $\mathrm{C} 12 \mathrm{~A} / \mathrm{C} 15 \mathrm{~A}$ holo state $\left(79.1^{\circ} \mathrm{C}\right)$ is higher than that of the $\mathrm{C} 12 \mathrm{~A} / \mathrm{C} 15 \mathrm{~A}$ apo state $\left(73.8^{\circ} \mathrm{C}\right)$, but they are not largely different. By contrast, $T_{m}$ of the AA $c_{555}$ holo state $\left(129.7^{\circ} \mathrm{C}\right)$ is much higher than that of the AA $c_{555}$ apo state $\left(\sim 70^{\circ} \mathrm{C}\right)$. This result indicates that the thermal stability is enhanced by the heme binding in terms of the water entropy but the effect of covalent heme linkages seems to be even larger. On the basis of another experimental study, it has been pointed out that the conformational entropy of the denatured state is decreased by covalent heme linkages. ${ }^{57}$

\section{CONCLUDING REMARKS}

We have investigated the physical origins of the difference among PA $c_{551}, \mathrm{PH} c_{552}$, HT $c_{552}$, and AA $c_{555}$ in terms of the thermal stability. The four cytochromes $c$ share significantly high sequence homology and similar threedimensional folds, but their denaturation temperatures are considerably different. The water-entropy gain at $25^{\circ} \mathrm{C}$ upon the transition from an extended structure to the native structure, which is normalized by the number of residues, is treated as a measure of the thermal stability. The measure is calculated using a hybrid of the angle-dependent integral equation theory ${ }^{16-29}$ combined with the multipolar water model ${ }^{17,18}$ and the morphometric approach. ${ }^{30,31}$

For the holo states, the order of the measure is in accord with that of the denaturation temperature: PA $c_{551}<\mathrm{PH} c_{552}$ $<\mathrm{HT} c_{552}<\mathrm{AA} c_{555}$, though the differences among the values of the measure for PA $c_{551}, \mathrm{PH} c_{552}$, and $\mathrm{HT} c_{552}$ are rather small. At high temperatures, the small differences in the measure leads to significantly large differences in the denaturation temperature, which stems from the temperature dependence of the conformational-entropy loss upon protein folding (see Fig. 2). AA $c_{555}$ has the lowest packing efficiency of heme and the apo polypeptide with hololike structure, which is unfavorable for the water entropy. The highest stability of AA $c_{555}$ is ascribed primarily to the highest efficiency of sidechain packing of the apo polypeptide itself. The extra helix of AA $c_{555}$ contributes to the high stability through the high sidechain packing efficiency. However, the intramolecular hydrogen bonds in the extra helix do not lead to the high stability.
It is often claimed that the higher packing efficiency contributes to the higher thermal stability through intramolecular van der Waals attractive interactions. It should be noted, however, that a gain in the intramolecular attractive interactions upon folding accompanies a loss of protein-water van der Waals attractive interactions. They are rather compensating. At higher temperatures, due to more energetic thermal motion of water molecules, protein-water van der Waals attractive interactions are less stable and the gain in the intramolecular attractive interactions could be predominant. As argued in Sec. IV D, however, even the electrostatic term of the energetic component, which should be larger than the van der Waals attractive term, does not play essential roles in determining the thermal stability. The higher packing efficiency enhances the thermal stability primarily through the water-entropy effect.

For all the four cytochromes $c$, the measure in the case without heme is considerably smaller than that in the case with heme. The thermal stability is enhanced by the heme binding in terms of the water entropy. On the basis of the experimental observations, ${ }^{5}$ we also argue that due to covalent heme linkages, the number of accessible conformations of the denatured state is decreased by the steric hindrance of heme, and the conformational-entropy loss upon folding becomes smaller, leading to an enhancement of the thermal stability.

Overall, our theoretical results are quite consistent with the experimental observations ${ }^{3-6}$ : Among the four cytochromes $c$, AA $c_{555}$ has the highest thermal stability for the holo state; the thermal stability is enhanced by the heme binding; though in the apo states of PA $c_{551}, \mathrm{PH} c_{552}$, and HT $c_{552}$ almost all helices are collapsed at $25^{\circ} \mathrm{C}$, the $\alpha$-helix content of the apo state of AA $c_{555}$ is almost equal to that of the holo state; and the apo AA $c_{555}$ polypeptide can intrinsically form a hololike structure without the heme binding.

Water definitely plays essential roles in the thermal stability of a protein. However, it is difficult to extract the underlying picture of the water roles in experiments. A theoretical approach accounting for the polyatomic structure of a protein and employing a molecular model for water, by which the hydration of a protein can be explored in detail, provides a solution of this problem. To the best of our knowledge, this is the first time that the effects of heme on the thermal stability of cytochromes $c$ are successfully elucidated by statistical thermodynamics.

\section{ACKNOWLEDGMENTS}

The computer program for the morphometric approach was developed with R. Roth and Y. Harano. We thank K. Amano for his help in preparing Table I. This work was supported by Grants-in-Aid for Scientific Research on Innovative Areas (Nos. 20118004 and 20118005) from the Ministry of Education, Culture, Sports, Science and Technology of Japan, by the Grand Challenges in Next-Generation Integrated Nanoscience, MEXT, Japan, and by Kyoto University Global Center of Excellence (GCOE) of Energy Science.

\footnotetext{
${ }^{1}$ R. Jaenicke and G. Böhm, Curr. Opin. Struct. Biol. 8, 738 (1998).
}

${ }^{2}$ R. Jaenicke, J. Biotechnol. 79, 193 (2000). 
${ }^{3}$ S. Nakamura, S. Ichiki, H. Takashima, S. Uchiyama, J. Hasegawa, Y. Kobayashi, Y. Sambongi, and T. Ohkubo, Biochemistry 45, 6115 (2006).

${ }^{4}$ M. Obuchi, K. Kawahara, D. Motooka, S. Nakamura, M. Yamanaka, T. Takeda, S. Uchiyama, Y. Kobayashi, T. Ohkubo, and Y. Sambongi, Acta Crystallogr. D Boil. Crystallogr. 65, 804 (2009).

${ }^{5}$ M. Yamanaka, H. Mita, Y. Yamamoto, and Y. Sambongi, Biosci., Biotechnol., Biochem. 73, 2022 (2009).

${ }^{6}$ O. Daltrop, J. W. A. Allen, A. C. Willis, and S. J. Ferguson, Proc. Natl. Acad. Sci. U.S.A. 99, 7872 (2002).

${ }^{7}$ Y. Harano and M. Kinoshita, Chem. Phys. Lett. 399, 342 (2004).

${ }^{8}$ Y. Harano and M. Kinoshita, Biophys. J. 89, 2701 (2005).

${ }^{9}$ T. Yoshidome, M. Kinoshita, S. Hirota, N. Baden, and M. Terazima, J. Chem. Phys. 128, 225104 (2008).

${ }^{10}$ M. Kinoshita, Front. Biosci. 14, 3419 (2009).

${ }^{11}$ M. Kinoshita, Int. J. Mol. Sci. 10, 1064 (2009).

${ }^{12} \mathrm{~S}$. Yasuda, T. Yoshidome, H. Oshima, R. Kodama, Y. Harano, and M. Kinoshita, J. Chem. Phys. 132, 065105 (2010).

${ }^{13}$ W. Kauzmann, Adv. Protein Chem. 14, 1 (1959).

${ }^{14} \mathrm{~K}$. Amano, T. Yoshidome, K. Oda, Y. Harano, and M. Kinoshita, Chem. Phys. Lett. 474, 190 (2009).

${ }^{15}$ S. Adinolfi, M. Nair, A. Politou, E. Bayer, S. Martin, P. Temussi, and A. Pastore, Biochemistry 43, 6511 (2004).

${ }^{16}$ J.-P. Hansen and I. R. McDonald, Theory of Simple Liquids, 3rd ed. (Academic, London, 2005).

${ }^{17}$ P. G. Kusalik and G. N. Patey, J. Chem. Phys. 88, 7715 (1988).

${ }^{18}$ P. G. Kusalik and G. N. Patey, Mol. Phys. 65, 1105 (1988).

${ }^{19}$ M. Kinoshita and M. Harada, Mol. Phys. 81, 1473 (1994).

${ }^{20}$ M. Kinoshita, S. Iba, and M. Harada, J. Chem. Phys. 105, 2487 (1996).

${ }^{21}$ M. Kinoshita and D. R. Bérard, J. Comput. Phys. 124, 230 (1996).

${ }^{22}$ N. M. Cann and G. N. Patey, J. Chem. Phys. 106, 8165 (1997).

${ }^{23}$ D. R. Bérard, M. Kinoshita, N. M. Cann, and G. N. Patey, J. Chem. Phys. 107, 4719 (1997).

${ }^{24}$ M. Kinoshita, J. Solution Chem. 33, 661 (2004).

${ }^{25}$ M. Kinoshita, J. Mol. Liq. 119, 47 (2005).

${ }^{26}$ M. Kinoshita, N. Matubayasi, Y. Harano, and M. Nakahara, J. Chem. Phys. 124, 024512 (2006).

${ }^{27}$ M. Kinoshita, J. Chem. Phys. 128, 024507 (2008).

${ }^{28}$ M. Kinoshita and M. Suzuki, J. Chem. Phys. 130, 014707 (2009).

${ }^{29}$ M. Kinoshita and T. Yoshidome, J. Chem. Phys. 130, 144705 (2009).

${ }^{30}$ P. M. König, R. Roth, and K. R. Mecke, Phys. Rev. Lett. 93, 160601 (2004).

${ }^{31}$ R. Roth, Y. Harano, and M. Kinoshita, Phys. Rev. Lett. 97, 078101 (2006).

${ }^{32}$ T. Imai, Y. Harano, M. Kinoshita, A. Kovalenko, and F. Hirata, J. Chem. Phys. 125, 024911 (2006).
${ }^{33}$ B. R. Brooks, R. E. Bruccoleri, B. D. Olafson, D. J. States, S. Swaminathan, and M. Karplus, J. Comput. Chem. 4, 187 (1983).

${ }^{34}$ A. D. MacKerell, Jr., D. Bashford, M. Bellott, R. L. Dunbrack, Jr., J. D. Evanseck, M. J. Field, S. Fischer, J. Gao, H. Guo, S. Ha, D. Joseph-McCarthy, L. Kuchnir, K. Kuczera, F. T. K. Lau, C. Mattos, S. Michnick, T. Ngo, D. T. Nguyen, B. Prodhom, W. E. Reiher III, B. Roux, M. Schlenkrich, J. C. Smith, R. Stote, J. Straub, M. Watanabe, J. Wiorkiewicz-Kuczera, D. Yin, and M. Karplus, J. Phys. Chem. B 102, 3586 (1998).

${ }^{35}$ A. D. Mackerell, Jr., M. Feig, C. L. Brooks III, J. Comput. Chem. 25, 1400 (2004).

${ }^{36}$ M. S. Lee, F. R. Salsbury, Jr., and C. L. Brooks III, J. Chem. Phys. 116, 10606 (2002).

${ }^{37}$ M. S. Lee, M. Feig, F. R. Salsbury, Jr., and C. L. Brooks III, J. Comput. Chem. 24, 1348 (2003).

${ }^{38}$ J. Chocholoušová and M. Feig, J. Comput. Chem. 27, 719 (2006).

${ }^{39}$ F. Autenrieth, E. Tajkhorshid, J. Baudry, and Z. Luthey-Schulten, J. Comput. Chem. 25, 1613 (2004).

${ }^{40}$ R. L. Dunbrack, Jr. and F. E. Cohen, Protein Sci. 6, 1661 (1997).

${ }^{41}$ R. L. Dunbrack, Jr., Curr. Opin. Struct. Biol. 12, 431 (2002).

${ }^{42}$ I. K. McDonald and J. M. Thornton, J. Mol. Biol. 238, 777 (1994).

${ }^{43}$ M. Feig, J. Karanicolas, and C. L. Brooks III, J. Mol. Graphics 22377 (2004).

${ }_{44}^{4}$ J. W. Ponder and F. M. Richards, J. Comput. Chem. 8, 1016 (1987).

${ }^{45}$ M. Ikeguchi and J. Doi, J. Chem. Phys. 103, 5011 (1995).

${ }^{46}$ M. Kinoshita, J. Chem. Phys. 116, 3493 (2002).

${ }^{47}$ K. Amano and M. Kinoshita, Chem. Phys. Lett. 488, 1 (2010).

${ }^{48}$ Y. Harano, T. Yoshidome, and M. Kinoshita, J. Chem. Phys. 129, 145103 (2008).

${ }^{49}$ T. Yoshidome, Y. Harano, and M. Kinoshita, Phys. Rev. E 79, 011912 (2009).

${ }^{50}$ T. Yoshidome and M. Kinoshita, Phys. Rev. E 79, 030905(R) (2009).

${ }^{51}$ H. Oshima, T. Yoshidome, K. Amano, and M. Kinoshita, J. Chem. Phys. 131, 205102 (2009).

${ }^{52}$ Y. Harano, R. Roth, and M. Kinoshita, Chem. Phys. Lett. 432, 275 (2006).

${ }^{53}$ Y. Harano, R. Roth, Y. Sugita, M. Ikeguchi, and M. Kinoshita, Chem. Phys. Lett. 437, 112 (2007).

${ }^{54}$ T. Yoshidome, K. Oda, Y. Harano, R. Roth, Y. Sugita, M. Ikeguchi, and M. Kinoshita, Proteins 77, 950 (2009).

${ }^{55}$ J. Fitter, Biophys. J. 84, 3924 (2003).

${ }^{56}$ N. Baden, S. Hirota, T. Takabe, N. Funasaki, and M. Terazima, J. Chem. Phys. 127, 175103 (2007).

${ }^{57}$ F. Arnesano, L. Banci, I. Bertini, and S. Ciofi-Baffoni, Biochemistry 39, 1499 (2000) 